\title{
Malathion degradation by Bacillus spp. isolated from soil
}

\author{
A.Ratna Kumari ${ }^{1 *}$, G. Jeevan ${ }^{1}$, M. Ashok ${ }^{1}$, Ch.Koteswara Rao ${ }^{2}$ K. S. K. Vamsi ${ }^{1}$ \\ 1. Department of Biotechnology, Bapatla Engineering College, Bapatla-522 101, A.P., India \\ 2. Centre for Biotechnology, Department of Chemical Engineering, Andhra University, Vizag-530 003, A.P., \\ India
}

Abstract-Organophosphate like Malathion is used for control of household and agricultural pests. High levels of malathion contaminates soil, water and aquatic ecosystems. Present study reports the isolation, morphological and biochemical analysis of malathion degrading bacteria. The analysis shows that isolated organism belongs to Bacillus species. The isolated organism was added to the soil supplemented with malathion \& incubated for 4 days at $37^{\circ} \mathrm{C}$ on shaker at 200rpm. After 4 days of incubation the phosphate levels are estimated by Fiske-Subbaraw method. The phosphate levels are increased due to degradation of malathion.

Keywords-Bacillus species, Fiske subbarow method, Malathion, Organophosphate.

\section{INTRODUCTION}

Currently among the various groups of pesticides that are being used the world over, organophosphorous group forms a major and most widely used group accounting for more than $36 \%$ of the total world market. Malathion [s-(1,2 -dicarbethoxyethyl)-o,o-dimethldithiophosphate), is an organophosphate pesticide widely used in public recreation areas, residential landscaping, and agricultural settings [1]. Malathion itself is of low toxicity, it mainly concentrates in peel and may not readily removed by washing in water alone [2] but easily enter the body through ingestion, inhalation and absorption through the skin results in its metabolism to malaoxon which is substantially more toxic [3]. Long-term exposure to oral ingestion of malaoxon in rats, showed 61 times more toxic than malathion. It is cleared from the body quickly, in three to five days [4]. Malathion degradation products include dimethyl phosphate, dimethyldithiophosphate, dimethylthiophosphate, isomalathion, malaoxon and due to cutinase, carboxylesterase, phosphatase enzymatic activity, malathion is degraded into malathion mono and dicarboxylic acid [1]. Malathion kills insects and other animals, including humans, by inhibiting the acetylcholinesterases (AChE) that breaks down acetylcholine a chemical essential in transmitting nerve impulses across junctions between nerves, thus prolonging action potential in nerves, causing spasma, incoordination, convulsions, paralysis and ultimately death. The World Health Organization estimates that 500,000 pesticides poisoning cases occur annually in the world and that $1 \%$ are fatal (5000 death/year) [5]. According to the Ministry of Commerce, Government of India, malathion residues were found exceeding the MRL (5 ppb) [6].

Microorganisms are used in bioremediation of environmental pollutants. Bacteria [7] and fungi [8] are capable of degrading malathion. Malathion can be degraded or detoxified using physical, chemical or biological methods. Using microorganisms is natural process, very effective when compared to the other methods and applicable for insitu bioremediation. Biodegradation of organophosphate compounds releases phosphate as one of the end product [9]. The present study describes the isolation of bacteria from malathion contaminated soils. Usually an unknown bacterium is identified by a combination of information obtained from microscopic observations like morphology, growth characteristics on agar, gram stain and other staining reaction, the absence or presence of motility, and biochemical tests.

After Morphological and biochemical analysis, showed that the bacteria belongs to Bacillus spp. group and is capable of degrading malathion.

\section{MATERIALS AND METHODS}

\subsection{Isolation of Bacteria from soil sample}

Malathion contaminated soil was obtained from different locations in and around Bapatla region. The collected soil sample is serially diluted and autoclaved. For isolation and selection of malathion degrading bacteria, LB Agar medium is prepared to this $100 \mu$ l of Dapsome (fungicide, to avoid the growth of fungi) is added. From each diluted sample $0.5 \mathrm{ml}$ was spread over the surface of petriplate with LB agar medium with the help of a glass spreader. The inoculated plates were subsequently incubated at $37^{\circ} \mathrm{C}$ for $48 \mathrm{hrs}$. Microbial colonies obtained were sub cultured for 4-6 times to obtain pure cultures. The colonies were isolated on the basis of the clear zone produced by the organism. 


\subsection{Identification of an isolated bacterial culture}

To identify the isolated bacterial culture Morphological analysis, cultural characteristics on agar plates $\&$ biochemical tests were performed. The morphological analysis comprises shape, motility \& Gram staining. The cultural characteristics include nutrition type, colony size, form, Growth on agar medium, optimum temperature. In order to observe biochemical activities of microorganisms which were specific to individual genus and species various kinds of specially prepared media were inoculated with pure cultures of isolated organisms. Many distinctive enzyme activities can be demonstrated by observing for the by products resulting from the action of enzymes on specific substrates within the specially prepared media. Different mediums were used for the biochemical characterization of the isolated and selected bacteria for their identification according to Bergey's Manual of Determinative Bacteriology. The biochemical analysis includes the Glucose fermentation (oxidation \& fermentation of glucose), Starch hydrolysis (Amylase production test), Hydrolysis of gelatin, Casein hydrolysis, Hydrogen sulfide production, Indole production test, Methyl red and Voges proskauer test, Citrate utilization, Urease test, Catalase test, Cellulase production test.

Carbohydrate catabolism by microorganism is performed to know the capacity of microorganisms to oxidize or fermentate glucose. Hugh and Leifson's medium (

$0.2 \mathrm{~g}$ peptone, $0.5 \mathrm{~g}$ sodium chloride, $0.03 \mathrm{~g}$ di-potassium hydrogen phosphate,

$1.5 \mathrm{ml}$ bromothymol blue solution, $0.3 \mathrm{~g}$ agar in $100 \mathrm{ml}$ of distilled water, $\mathrm{pH}$ 7.1) was prepared [10]. Inoculate two tubes of medium with the isolated microorganism by stabbing with a straight wire. Pour liquid paraffin over the medium to form a layer about one $\mathrm{cm}$ in depth into one of the tubes and the other one open to the air. Two uninoculated tubes may be used as control. Incubate the tubes at $35^{\circ} \mathrm{C}$ for $24-48$ hours. Microorganisms use carbohydrate differently depending upon their complement. The type of fermentation is characteristic of certain species, genera, or groups of organisms so this reaction is used as a method for identifying an unknown organism. Glucose in the cell can be catabolized either aerobically, anaerobically or by both. The end products of carbohydrate fermentation can be either organic acids (e.g. lactic, formic or acetic) or organic acid and gas (e.g. hydrogen or carbon dioxide). If the organism utilises trypton produces a dark blue colour which is an alkaline reaction, or if it utilises glucose produces acid and it turns the medium from blue to yellow. If the microorganism is able to produce acid in both closed and open tubes are termed as fermentative while those which produce acid only in the open tube are called oxidative.

Starch hydrolysis test was performed to determine the capability of microorganisms to use starch in the media. Starch agar media $(0.5 \mathrm{~g}$ peptone, $0.2 \mathrm{~g}$ starch, $0.3 \mathrm{~g}$ beef extract, $1.5 \mathrm{~g}$ agar in $100 \mathrm{ml}$ of distilled water, $\mathrm{pH}$ 7.0) was prepared [10]. Inoculate with the isolated microorganism and incubate for $48 \mathrm{hrs}$ at $37^{\circ} \mathrm{C}$. In presence of starch, the production of extracellular enzymes like amylase occurs indicating the potential of the organism to use starch as carbon source. To determine the presence or absence of starch in the medium iodine solution is used as an indicator. Few drops of iodine solution $(0.1 \mathrm{~N})$ were poured on $48 \mathrm{hrs}$ old cultures grown on Petri-plates and observations were made. Formation of blue color indicates the absence of potential to utilize starch by the organism. Formation of yellow zone around a colony indicates the hydrolysis of starch.

Gelatin hydrolysis test was performed to determine capability of microorganisms to use gelatin as media. Degradation of gelatin indicates the presence of gelatinase enzyme [10]. The actively grown isolated cultures are inoculated in nutrient gelatin medium $(0.25 \mathrm{~g}$ peptone, $0.15 \mathrm{~g}$ beef extract, $0.6 \mathrm{~g}$ gelatin in $100 \mathrm{ml}$ of distilled water) and grown for 4 to 7days. After incubation, the growing cultures are subjected to low temperature at $4^{0} \mathrm{C}$ for $15 \mathrm{~min}$. Cultures which have gelatinase producing capability degrades the gelatin and liquefies it shows positive test for gelatin hydrolysis. And those that remain solid indicate a negative reaction for gelatin hydrolysis.

Casein hydrolysis can be done by supplementing nutrient agar medium with milk [10]. The medium is opaque due to the presence of casein in colloidal suspension. Inoculate the skim milk agar plates (10g skim milk powder, $0.5 \mathrm{~g}$ peptone, $1.5 \mathrm{~g}$ agar $100 \mathrm{ml}$ of distilled water, $\mathrm{pH}$ 7.2) with the isolated microorganism and incubate for 24 to $48 \mathrm{hrs}$ at $37^{\circ} \mathrm{C}$ in an inverted position. Formation of a clear zone adjacent to the bacterial growth, after incubation, is a positive reaction for casein hydrolysis. While the absence of clear zone around the organism is a negative reaction.

The hydrogen sulfide production can be detected by incorporating a heavy metal salt containing $\left(\mathrm{Fe}^{2+}\right)$ or lead $\left(\mathrm{Pb}^{2+}\right)$ ion as $\mathrm{H}_{2} \mathrm{~S}$ indicator to a nutrient culture medium containing cysteine and sodium thiosulfate as the sulfur substrate [10]. Sulfide indole motility (SIM) agar media (30g peptone, $3 \mathrm{~g}$ beef extract, $0.2 \mathrm{~g}$ ferrous ammonium sulphate, $0.0025 \mathrm{~g}$ sodium thiosulphate, $3.0 \mathrm{~g}$ agar in $100 \mathrm{ml}$ of distilled water, $\mathrm{pH} 7.3$ ) was prepared. Inoculate the sulfide indole agar plates with the isolated microorganism and incubate for $48 \mathrm{hrs}$ at 35 to $36^{\circ} \mathrm{C}$. When hydrogen sulfide, a colourless gas, is produced it reacts with the metal salt (ferrous sulfates) and forms a visible insoluble black ferrous sulfide precipitates. It indicates the positive test for hydrogen sulphide production. The absence of black coloration indicates negative test.

The indole test is performed by inoculating isolated bacterium into $1 \%$ tryptone broth ( $1 \mathrm{~g}$ of peptone in $100 \mathrm{ml}$ of distilled water) and incubating at $35^{\circ} \mathrm{C}$ for $48 \mathrm{hrs}$ [10]. After incubation, the indole produced is detected 
by adding Kovac's reagent (dimethylaminobenzaldehyde) which produces a cherry red reagent layer as illustrated:

Tryptophan $\longrightarrow$ Indole + pyruvic acid + ammonia

Indole $+\mathrm{p}-$ dimethylaminobenzaldehyde $\longrightarrow$ Cherry red compound

The development of cherry red color in the top layer of the tube is a positive test for indole production. Absence of red coloration is an indole negative test.

The methyl red (MR) and the voges-proskauer (VP) tests are used to differentiate two major types of bacteria like Escherichia coli and Enterobacter aerogenes. MR-VP broth $(0.7 \mathrm{~g}$ peptone, $0.5 \mathrm{~g}$ dextrose, $0.5 \mathrm{~g}$ potassium phosphate in $100 \mathrm{ml}$ of distilled water, $\mathrm{pH}$ 6.9) was prepared [10]. Inoculate the MR-VP tubes with the isolated microorganism and incubate for $48 \mathrm{hrs}$ at $35^{\circ} \mathrm{C}$ and a control was maintained. Add 5 drops of methyl red indicator to one set of tubes. In these tests, if an organism produces large amount of organic acids from dextrose, after the addition of methyl red a $\mathrm{pH}$ indicator (i.e.,pH remaining below 4.4), the medium will remain red which indicates a positive test. In some organisms methyl red will turn to yellow, which indicates a negative test. It is due to the elevation of $\mathrm{pH}$ above 6.0 because of the enzymatic conversion of the organic acids (produced during the glucose fermentation) to nonacidic end products such as ethanol and acetoin. To the other set of tubes add 12drops of VP reagent I \& II. Shake the tubes gently for $30 \mathrm{sec}$ by exposing the medium to oxygen and allow for 15-30min completing the reaction. The development of crimson to ruby pink colour is an indicative of the positive reaction and no change in color is a negative reaction.

The citrate test is done by inoculating the isolated organism into an organic synthetic medium, simmon's citrate agar (0.1g Ammonium dihydrogen phosphate, 0.1g Dipotassium phosphate, $0.5 \mathrm{~g}$ Sodium chloride, $0.2 \mathrm{~g}$ sodium citrate, $0.02 \mathrm{~g}$ Magnesium sulphate, $1.5 \mathrm{~g}$ agar, $0.08 \mathrm{~g}$ bromothymol blue in $100 \mathrm{ml}$ of distilled water, $\mathrm{pH}$ 6.9) and incubate for $48 \mathrm{hrs}$ at $37^{\circ} \mathrm{C}$ [10]. If the citric acid is metabolised it generates the $\mathrm{CO}_{2}$. The generated $\mathrm{CO}_{2}$ combines with sodium and water forms an alkaline sodium carbonate, which changes the colour of the bromothymol blue from green to blue and this, constitutes a positive test. If the medium remains green in color indicates citrate negative.

Urea is one of the major organic waste products of protein digestion in most vertebrates and is excreted in the urine. Some microorganisms have the ability to produce the enzyme urease. The urease is a hydrolytic enzyme which breaks the carbon and nitrogen bond in amide compounds, liberates ammonia.

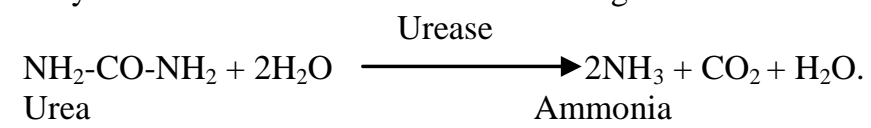

To perform urease test, urea agar medium $(0.1 \mathrm{~g}$ peptone, $0.5 \mathrm{~g}$ sodium chloride, $0.2 \mathrm{~g}$ potassium monohydrogen, $0.2 \mathrm{~g}$ agar in $100 \mathrm{ml}$ of distilled water, $\mathrm{pH} 6.8$ after autoclave add $0.1 \mathrm{~g}$ glucose and $0.6 \mathrm{ml}$ of phenol red steam for $1 \mathrm{hr}$, cool to $50^{\circ} \mathrm{c}$, add $20 \%$ urea and sterilize by filtration)was prepared [10]. Inoculate the isolated microorganism in urea agar medium. Incubate for 24 to 48 hours at $37^{\circ} \mathrm{C}$. During incubation, microorganisms which have the capability to produce urease will liberate ammonia that raises the $\mathrm{pH}$ of the medium. The rise in $\mathrm{pH}$ changes the phenol red color from yellow (pH6.8) to a red or deep pink. No change in color is an evidence of a lack of urease production by the microorganisms.

Catalase test is performed using Hydrogen peroxide and trypticase soy agar medium. Trypticase soy agar medium ( $1.5 \mathrm{~g}$ trypticase, $0.5 \mathrm{~g}$ phytone, $0.5 \mathrm{~g}$ sodium chloride, $1.5 \mathrm{~g}$ agar in $100 \mathrm{ml}$ of distilled water, $\mathrm{pH} 7.3$ ) was prepared. Inoculate the medium with the isolated organism and incubate for $48 \mathrm{hrs}$ at $35^{\circ} \mathrm{C}$ [10]. After incubation, add 3-4 drops of hydrogen peroxide by holding the tube at an angle. Catalase enzyme is present in microorganisms breaks down hydrogen peroxide to water and oxygen. Release of oxygen gas forms bubbles it indicates catalase positive test and no bubble formation indicate catalase negative.

Cellulase test, evidence for the microbial utilization of cellulose can be detected using hexadecyltrimethyl ammonium bromide. This reagent precipitates intact carboxymethyl cellulose in the medium and thus clear zones around a colony in an otherwise opaque medium indicating degradation of CMC. To perform cellulase test Czapek-mineral salt agar medium $(0.2 \mathrm{~g}$ sodium nitrate, $0.1 \mathrm{~g}$ potassium phosphate, $0.05 \mathrm{~g}$ Magnesium sulphate, $0.5 \mathrm{~g}$ Potassium chloride, $0.2 \mathrm{~g}$ peptone, $2 \mathrm{~g}$ agar in $100 \mathrm{ml}$ of distilled water, $\mathrm{pH} 6.5$ ) was prepared [10]. Inoculate the czapek-mineral salt agar medium with the isolated microorganism. Incubate at $35^{\circ} \mathrm{C}$ in an inverted position for 2-5days. Flood the incubated plates with $1 \%$ aqueous solution of hexadecyltrimethyl ammonium bromide. Formation of a clear zone around the growth of the microorganism indicates the degradation of carboxymethyl cellulose by extracellular cellulose.

\subsection{Analysis of malathion degradation and estimation of released phosphate by Fiske Subbaraw method}

For analysis of malathion degradation, the isolated microorganism is incubated with malathion at $37^{\circ} \mathrm{C}$ for 4 days on a shaker at 200rpm. At the same time control was maintained without inoculums. As a control malathion free soil sample was taken and for test malathion was added to the soil sample. After 4 days of 
incubation the phosphate levels were estimated using Fiske-Subbaraw method [11]. From the standards, the phosphorus content present in the soil supplemented with malathion were measured.

\section{III.RESULTS}

Pure cultures were obtained on medium after incubation at $37^{\circ} \mathrm{C}$ after $48 \mathrm{hrs}$. The morphological characteristics include shape, motility \& Gram staining. The isolated organism were large, able to grow at optimum temperature, showed heterotrophic type of nutrition, rod shaped structure at one end it contains an oval endospore, motile and Gram positive as shown in Fig-1. For the biochemical analysis the isolated organism showed positive response for the Starch hydrolysis, Hydrolysis of gelatin, Casein hydrolysis, Catalase test. After performing starch hydrolysis test, a yellow zone is formed indicates the hydrolysis of starch as shown in Fig-2. In gelatin test the cultures are able to produce the gelatinase, degraded the gelatin so that the medium was liquefied as shown in Fig-3. In casein hydrolysis a clear zone was formed adjacent to the bacterial culture as shown in Fig-4. Oxygen bubbles are formed in a positive catalase test as shown in Fig-5. During the analysis of malathion degradation, the isolated microorganism showed an increase in phosphorous content by the FiskeSubbaraw method.

\section{DISCUSSION}

Agricultural productivity is increased by using pesticides, but only less percentage of pesticide is sufficient to kill the pesticide remaining pesticide causes different types of pollutions. Different species of Pseudomonas $[12,13,14,15,16]$ and the fungal species like Aspergillus oryzae [17] are able to degrade the malathion. Microorganisms play an important role in the detxoificaiton, keeping in view of this microorganism was isolated from the malathion contaminated soil. To identify this unknown culture, first the genus to which it fits should know. The genus is identified using the Table -1 [10]. Based on morphological, cultural and biochemical characteristics the isolated bacteria were rod shaped, Gram positive, able to form endospore and showed positive response to the starch hydrolysis, gelatin hydrolysis, casein hydrolysis, catalase test. As shown in Table-1 Gram positive, endospore forming bacteria are Bacillus, Clostridium, Sporolactobacillus. Out of these three, only Bacillus shows positive response to the catalase test. With these the bacteria isolated from soil was identified as species of the genus Bacillus. The Fiske-Subbaraw method indicates that the amount of phosphate was increased in the sample which was treated with the isolated Bacillus bacteria, it is due to the detoxification of malathion. So, with this test it is confirm that the organism which was isolated is capable of degrading malathion.

\section{CONCLUSION}

The study shows that the isolated bacterium is able to degrade the malathion, so that this organism is used as a biological agent for the insitu bioremediation of malathion contaminated soil. The present study is done only on the morphological, cultural and biochemical characterisation of isolated bacteria. Further characterisation like 16s rRNA analysis and sequencing of the organophosphate degrading gene is required and is going on. Instead of using the chemical methods for degrading pesticides, these ecofriendly bacteria can be used and motivate the farmers to use natural biological pesticides.

\section{REFERENCES}

[1]. B. K. Singh, A. Walker, Microbial degrading of organophosphorus compounds, FEMS Microbiol Rev., 30: 2006, 428-471

[2]. G. Nath, M.K. Srivastava, Effect of processing on the removal of malathion from treated cabbages (Brassica oleracea L.var. capitata), Indian J. Ent. 52:1990, 300-309.

[3]. D. Edwards, "Reregistration Eligibility Decision for Malathion", US Environmental Protection Agency - Prevention, Pesticides and Toxic Substances EPA 738-R-06-030 journal: 9. 2006.

[4]. Maugh II, H. Thomas H, "Study links pesticide to ADHD in children", Los Angeles Times. http://articles.latimes.com/2010/may/16/science/la-sci-pesticides-20100517. 16 May 2010.

[5]. L.M. Rosenstock, W.E.Keifer, R. Daniell McConnel, K. Claypoole, Chronic central nervous system effects of acute organophosphate pesticide intoxication, Lancet 338: 1991, 223-227.

[6]. SK. Goda SK, Iman E Elsayed, TA. Khodair, Walaa El-Sayed, ME. Mohamed, Screening for and isolation and identification of malathion-degrading bacteria: cloning and sequencing a gene that potentially encodes the malathion-degrading enzyme, carboxylestrase in soil bacteria, Biodegradation 21(6), 2010, 903-913.

[7]. B. Singh, J. Kaur, K. Singh, Biodegradation of malathion by Brevibacillus sp. strain KB2 and Bacillus cereus strain PU, World Journal of Microbiology \& Biotechnology 28(3), 2012, 1133-1141.

[8]. Chalamala Ramadevi, Mitta Mahendra Nath, Muppala Guru Prasad, Mycodegradation of malathion by a soil fungal isolate, Aspergillus niger, International Journal of Basic and Applied Chemical Sciences. Vol. 2 (1), 2012, 108-115

[9]. Pradnya P Kanekar, Bharati J Bhadbhade, Neelima M Deshpande, Seema S Sarnail, Biodegradation of Organophosphorus Pesticides, Proc. Indian natn Sci Acad. B70 No. 1; 2004, 57- 70.

[10]. K.R.Aneja, Experiments in microbiology, plant pathology and biotechnology, (New age international publishers, fourth edition, 2003).

[11]. J. Jayaram, Laboratory manual in Biochemistry (New age international publishers; 2002, 103-104). 
[12]. A.W. Bourquin, Microbial malathion interaction in artificial salt marsh ecosystems - fect and degradation, EPA. Ecol. Res.Ser.41.1975.

[13]. A.W. Bourquin, Degradation of malathion by salt marsh microorganisms, Appl. Environ. Microbiol. 33: 1977, 356-362.

[14]. A. Rosenberg, M. Alexander, Microbial cleavage of various organophosphorous insecticides, Appl. Environ. Microbiol. 37: $1979,886-891$.

[15]. L.J.R. Foster, H. Bia, Microbila degradation of the organophosphate pesticide, Ethion. FEMS Microbiol. Lett.240: 2004, 49-53.

[16]. I. Hashmi, M.A. Khan, J.G. Kim, Malathion degradation by Pseudomonas using activated sludge treatment system (Biosimulator), Biotechnology 3: 2004, 82-89.

[17]. D.L. Lewis, D.F.Paris, G.L. Baughman, Transformation of malathion by a fungus Aspergillus oryzae isolated from a fresh water pond, Bull. Environ. Contam. Toxicol. 13: 1975, 596.

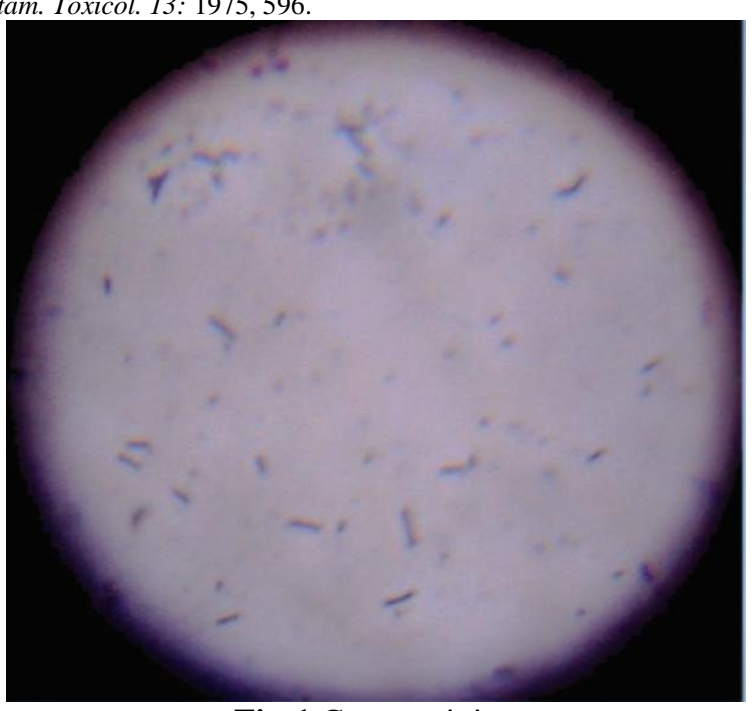

Fig-1 Gram staining

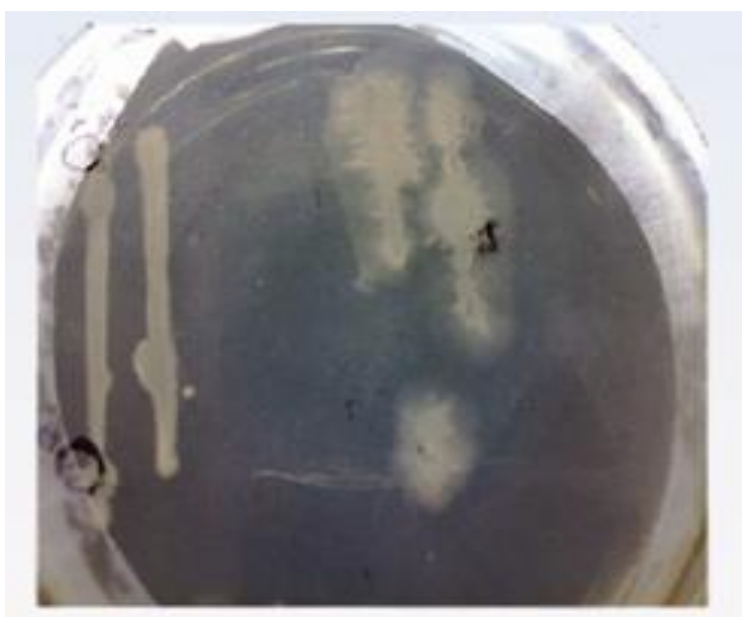

Fig-2 Starch hydrolysis

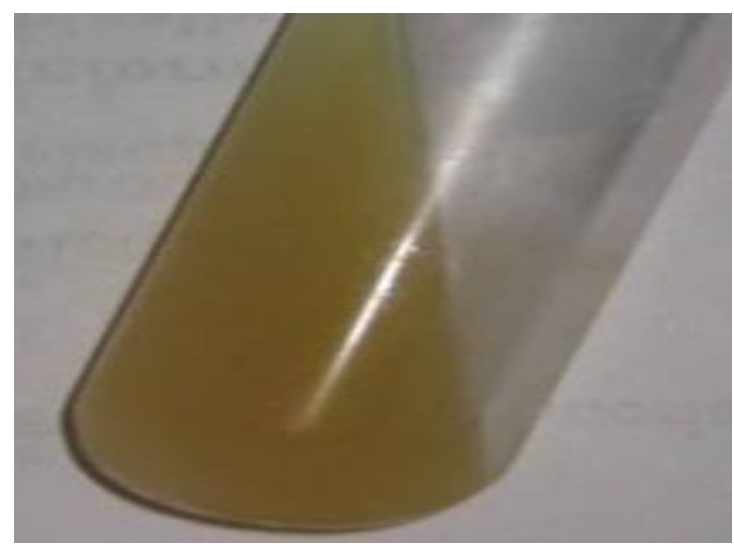

Fig-3 Gelatin hydrolysis 


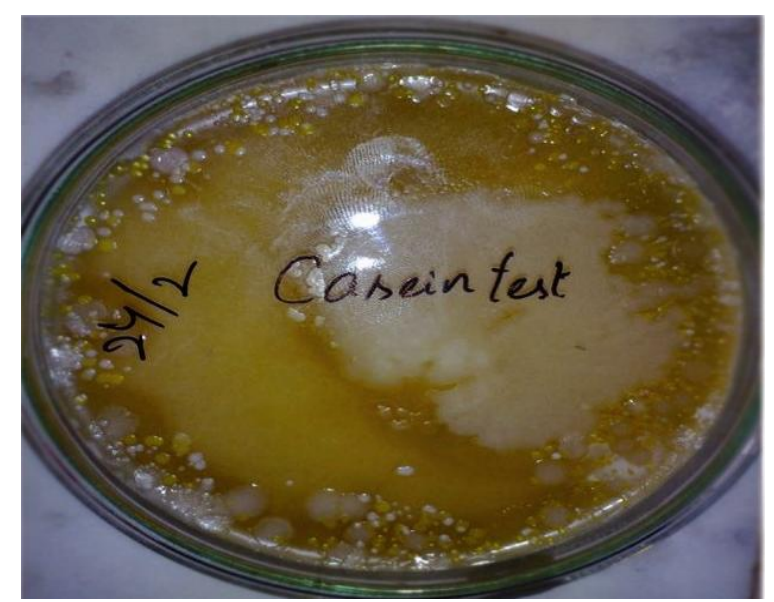

Fig-4 Casein hydrolysis

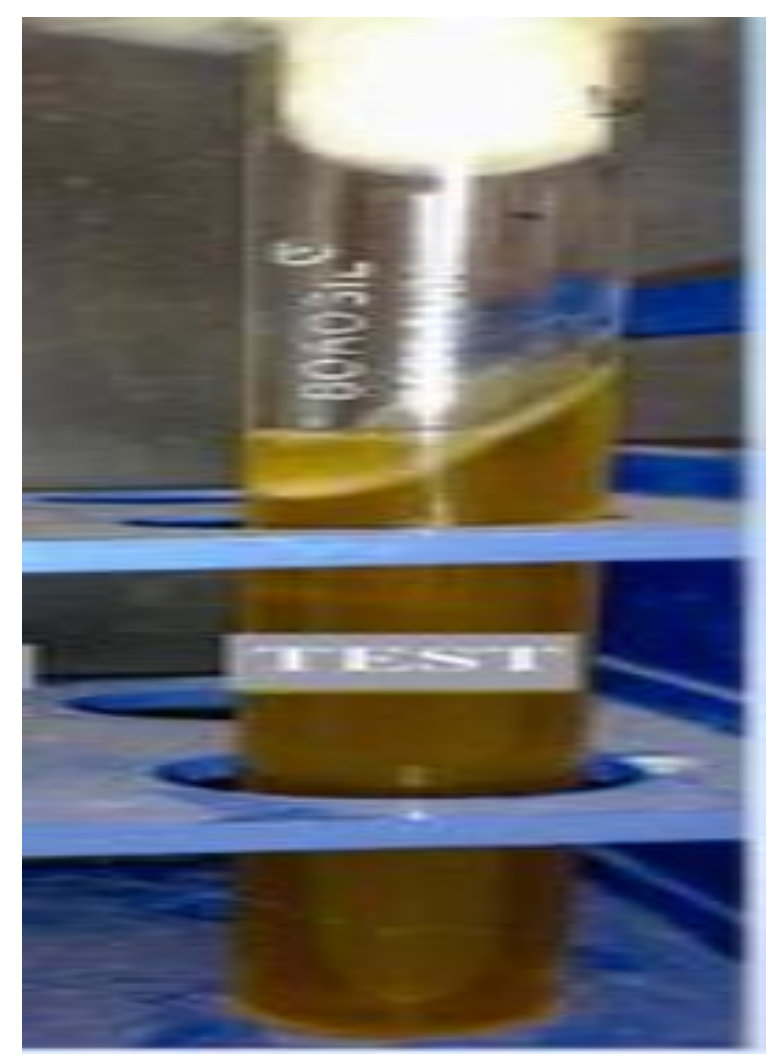

Fig-5 Catalase test

Table-1 Identification of a unknown bacterial culture upto genus level Rods and cocol

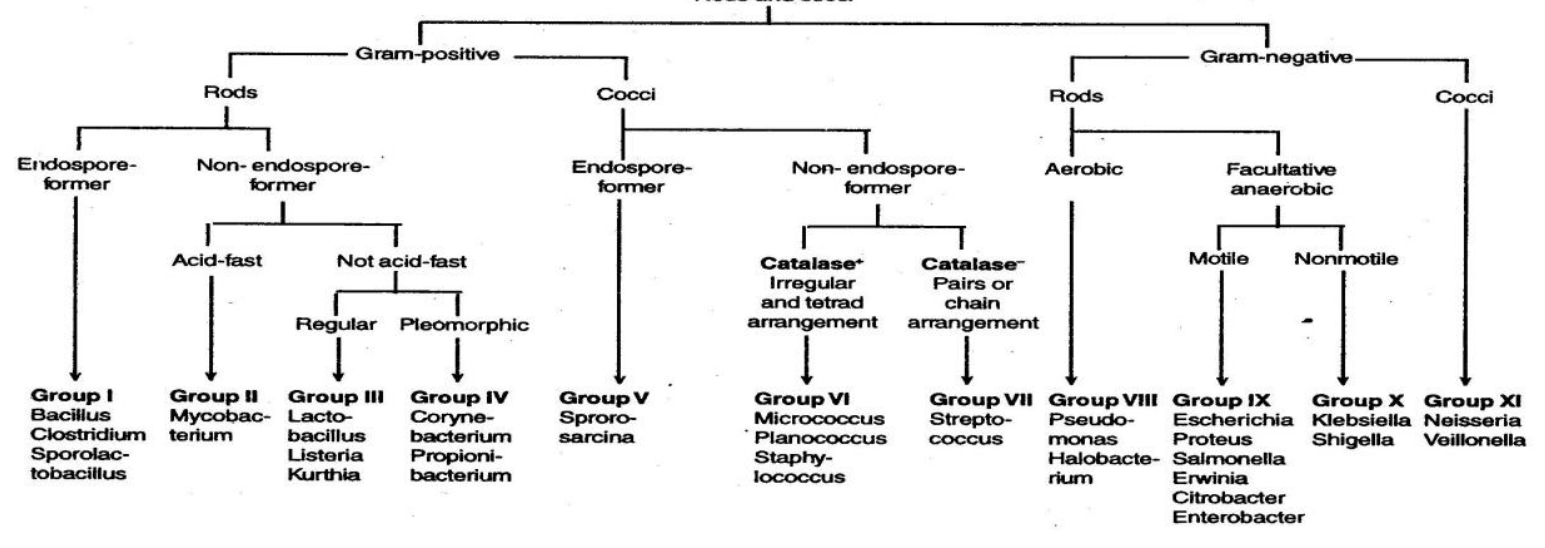

